\title{
Temporal Autocorrelation Properties of Three Dimensional Vehicle-to -Vehicle Channels in the Presence of Moving Scatterers
}

\author{
Xiaoping Zeng, Yu Zhou, Xin Jian, Jie Huang and Jing Li \\ College of Communication Engineering, Chongqing University, China \\ jianxin_zg@163.comorjianxin@cqu.edu.cn
}

\begin{abstract}
This paper derives the analytical expression of the temporal autocorrelation function $(A C F)$ of the non-stationary three dimensional (3D) multipath scattering channels, in which scatterers move with constant velocities in random directions around transceivers. The analytical expression involves high dimensional integration within finite interval, thus a methodology based on ray-tracing is proposed to carry out its numerical solution. The analytical expression and the corresponding ray-tracing based model serve as the canonical forms for the correlation analysis of non-stationary $3 D$ multipath scattering channels, which include two special cases: two dimensional (2D) multipath scattering and stationary multipath scattering. Numerical results show that: in case of nonstationary $3 D$ multipath scattering, with the increase of the velocity and the density of scatterers, the temporal ACF would attenuate more rapidly. These results would allow engineers more appropriate determination on coherence time and coherence bandwidth for vehicle-to-vehicle communication systems.
\end{abstract}

Keywords: 3D multipath scattering, Non-stationary multipath Scattering, Temporal autocorrelation function, Ray-tracing based method, vehicle-to-vehicle communication

\section{Introduction}

The rapid increase in road traffic density leads to congestion on streets and accidents, which brings serious threat to people's property and personal safety. Vehicle-to-vehicle (V2V) communication systems are a promising technology to alleviate or avoid these injuries because they have the potential to improve convenience and safety of traffic transportation [1]. V2V communication systems can find applications in cellular networks, intelligent transportation systems and future combat systems [2]. For the development of future V2V communication systems and standards, a deep knowledge of the underlying fading channel characteristics is of prime important.

In $\mathrm{V} 2 \mathrm{~V}$ communication systems, both the transmitters and receivers are in motion and surrounded by local scatterers. The channel characteristics of the V2V scenario are influenced by the typical traffic patterns and by the environment around the transceivers [2]. Different V2V models have been proposed to research the channel characteristics in the literatures. For 2D scenario, $[3,4]$ propose the geometric scattering model for streets and derive the cross-correlation function $(\mathrm{CCF})$ and temporal autocorrelation function (ACF). [5] proposes a two-ring model to describe the typical urban scattering scenario and derives the CCF of the complex channel gains. Combined two-ring model and ellipse model, [6] obtains the space-time-frequency correlation function (STF-CF) and power spectral density (PSD) for the non-isotropic Ricean fading channels. As special cases of V2V channels, fixed-to-vehicle (F2V) channels are analyzed in $[7,8]$, and the statistics of angle-of-arrival (AOA), angle-of-departure (AOD) and time-of-arrival (TOA) of the channel are studied. All the above models assume the scatterers around transceivers are stationary. However, non-stationary or moving scatterers are ubiquitous in $\mathrm{V} 2 \mathrm{~V}$ 
communication systems. The non-stationary scatterers may include walking pedestrians, moving foliage, and passing vehicles, which can be a significant source for the Doppler spread, particularly at millimeter wavelengths [9]. [9] considers the situation when moving scatterers concentrate on a ring centered on the fixed receiver for $2 \mathrm{D}$ fixed-tofixed (F2F) channels. [10] discusses the influence of moving scatters with deterministic velocities and deterministic directions on the CCF and ACF of 2D V2V channels. [11] and [12] derive the ACF, PSD and Doppler spread of 2D V2V channel in the presence of moving scatterers with random velocities and random directions analytically. However, all of these models restrict them to 2D multipath scattering without extending their results to the three dimensional (3D) cases, which is more opportune to model the movement of scatterers in real-world. Recently, with the development of intelligent transportation, more and more literatures dedicate their studies on the statistical behavior of 3D channels. [13] derives the closed-form expression of joint space-time correlation function (ST-CF) for 3D non-isotropic scattering scenario where scatterers are fixed on the surface of two cylinders. [14] and [15] propose a geometrical model where scatterers are limited in the volume of the two spheres, and deduce its space-time-frequency cross-correlation function (STF-CF) and ACF, respectively. [16] proposes a 3D semi-ellipsoidal model where scatterers are confined with the volume of the semi-ellipsoids, and studies the joint probability density function (PDF) of the azimuth and elevation AOD. However, all these 3D models suppose the scatterers are stationary. For non-stationay 3D case, [2] restrictes the scatterers in the volume of cylinders and derived the ST-CF and space-Doppler PSD of the channel. In this paper, we would analyze another non-stationary $3 \mathrm{D}$ multipath scattering scenario where the local scatterers are moving with constant velocities in random directions and no constrains are imposed on the position of the moving scatterers. To be more specific, we extend the results in [11] and [12] into its 3D case and derive the corresponding analytical expression of temporal ACF. As the analytical expression involves high dimensional integration, the corresponding numerical solution is given out by the usage of ray-tracing based model, with which the influences of different scatterer velocities and different scatterer densities on the ACF are discussed.

The remainder of this paper is organized as follows. Section 2 presents the analytical expression for the temporal ACF of non-stationary 3D multipath scattering. Section 3 introduces the ray-tracing based simulation model for numerical calculation. Section 4 presents the numerical simulation results and the related discussions. Section 5 summarizes the main contributions and concludes the paper.

\section{Theoretical Analysis}

\subsection{Non-Stationary Three Dimension (3D) Scattering Model}

The non-stationary scattering 3D model shown in Fig. 1 can appropriately model the typical propagation characteristics of urban areas, where transceivers are surrounded by $N_{S}$ local moving scatterers denoted by $S^{n}\left(n=1,2, \cdots, N_{S}\right)$. The main parameters of the $n$th path can be explained as follows.

1) The origin of the 3D Cartesian coordinate system is the initial position of receiver. The $\mathrm{x}$-axis is the line of sight (LOS) between receiver $C_{R}$ and transmitter $C_{T}$, and the positive direction of $\mathrm{x}$-axis is from the transmitter $C_{T}$ to the receiver $C_{R} . S^{n}$, is the position of the $n$th scatterer $S^{n}$ projected on the $\mathrm{x}-\mathrm{y}$ plane.

2) The transmitter and the receiver move with the constant velocities $v_{T}$ and $v_{R}$; the azimuth and elevation angle of the moving direction of transmitter are $\alpha_{T}$ and $\beta_{T}$; the azimuth and elevation angle of the moving direction of receiver are $\alpha_{T}$ and $\beta_{T}$, respectively. 
3) The azimuth and elevation AOD from transmitter $C_{T}$ to the $n$th scatterer $s^{n}$ are $\alpha_{T}^{n}$ and $\beta_{T}^{n}$; the azimuth and elevation AOA from the $n$th scatterer $S^{n}$ to the receiver $C_{R}$ are $\alpha_{R}^{n}$ and $\beta_{R}^{n}$, respectively.

4) The $n$th scatterer $S^{n}$ moves around transceivers with the constant velocity $v_{S^{n}}$, random azimuth angle $\alpha_{v}^{s^{n}}$ and random elevation angle $\beta_{v}^{s^{n}}$, which represent the non-stationary scattering caused by the random movement of scatterers.

5) The azimuth angle of LOS is $\alpha_{R}^{L O S}=\pi$.

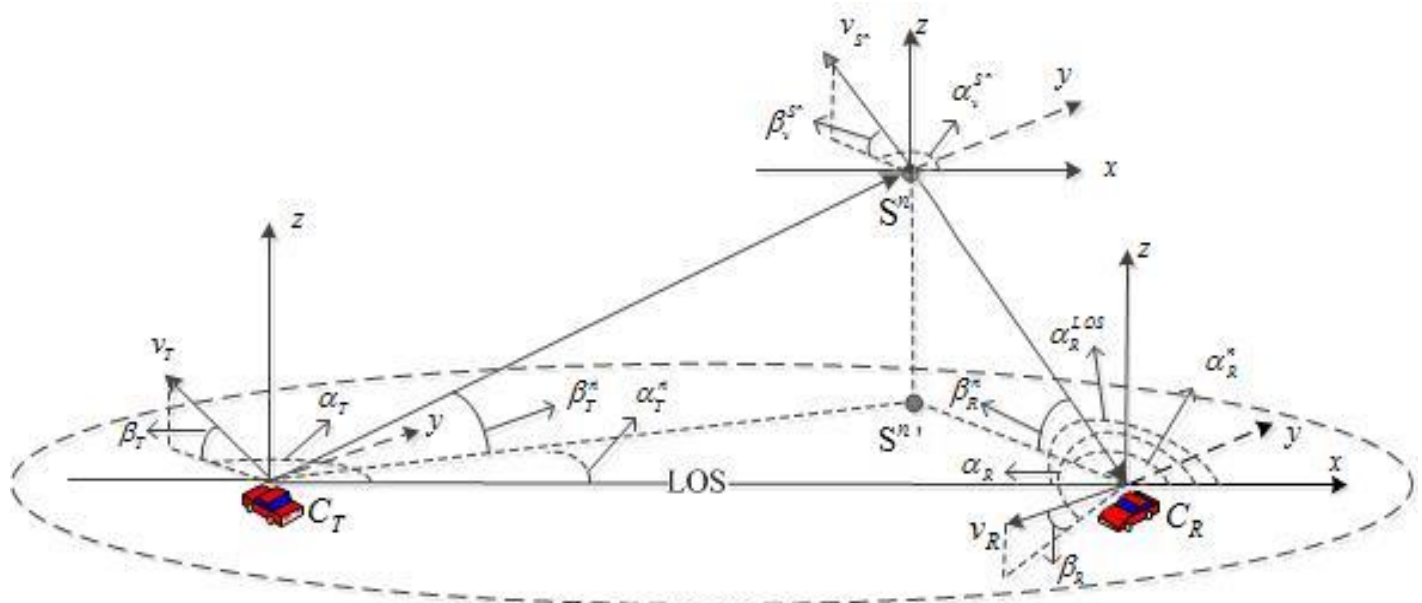

Figure 1. 3D Non- Sstationary Scattering Scenario

\subsection{ACF of Non-Stationary 3D Multipath Scattering}

The established non-stationary 3D scattering propagation model in Figure 1 is an extension of the 2D case analyzed in [11]. The complex channel gain of frequencynonselective F2V channels with stationary scatterers can be presented as [17]

$$
\mu(t)=\sum_{n=1}^{N_{s}} c_{n} e^{j\left(2 \pi f_{n} t+\theta_{n}\right)},
$$

where $c_{n}$ is the attenuation factor caused by the interaction of the emitted wave with the $n$th scatterer $S^{n}, f_{n}$ is the Doppler shift caused by the moving receiver, $\theta_{n}$ represents the phase shift of the $n$th path, which is not considered in this paper and $\theta_{n}=0$.

The channel impulse response described above cannot be used to model the 3D V2V propagation scenario in Fig. 1 directly. To make it tractable, the effects of both moving transmitter and moving scatterers have to be considered. According to [11], $f_{n}$ can be decomposed into four parts, namely ${f_{n}}^{T},{f_{n}}^{T S}, f_{n}{ }^{S R}$ and $f_{n}{ }^{R}$, and

$$
f_{n}=f_{n}^{T}+f_{n}^{T S}+f_{n}^{S R}+f_{n}^{R},
$$

in which, the first part of Doppler shift $f_{n}{ }^{T}$ is due to the movement of the transmitter; the second part $f_{n}{ }^{T S}$ is the contribution which arises when the transmitted electromagnetic signal impinges on the $n$th moving scatterer $S^{n}$; the 
third part $f_{n}{ }^{S R}$ is due to the fact that the moving scatterer redirects the signal to the receiver; the last part $f_{n}^{R}$ results from the moving receiver.

It is of prime important to find the expressions of $f_{n}{ }^{T}, f_{n}{ }^{T S}, f_{n}{ }^{S R}$ and $f_{n}{ }^{R}$ in 3D case, because the transmission path of electromagnetic wave is really $3 \mathrm{D}$ rather than 2D. As they share the similar deduction process, here we just illustrate the derivation process of $f_{n}{ }^{S R}$ for reference. The scattering path from the the $n$th scatterer $S^{n}$ to the receiver is illustrated in Fig. 2. The direction of AOA is $\left(\alpha_{R}^{n}, \beta_{R}^{n}\right)$, the velocity direction of scatterer is $\left(\alpha_{v}^{s^{n}}, \beta_{v}^{s^{n}}\right)$, and the Doppler shift can be shown as

$$
f_{n}^{S R}=\frac{v_{S^{n}}}{\lambda}\left[\cos \left(\alpha_{v}^{S^{n}}-\alpha_{R}^{n}\right) \cos \beta_{R}^{n} \cos \beta_{v}^{S^{n}}+\sin \beta_{v}^{S^{n}} \sin \beta_{R}^{n}\right],
$$

where $\lambda$ is the wavelength of the carrier, $\lambda=c / f_{c}$, and $c$ is the velocity of light. By setting $\beta_{R}^{n}=0, \beta_{T}^{n}=0$ and $\beta_{v}^{S^{n}}=0, f_{n}{ }^{S R}$ corresponds to its $2 \mathrm{D}$ case, that is, $f_{n}^{S R}=v_{S^{n}} / \lambda \times\left[\cos \left(\alpha_{v}^{S^{n}}-\alpha_{R}^{n}\right)\right]$.

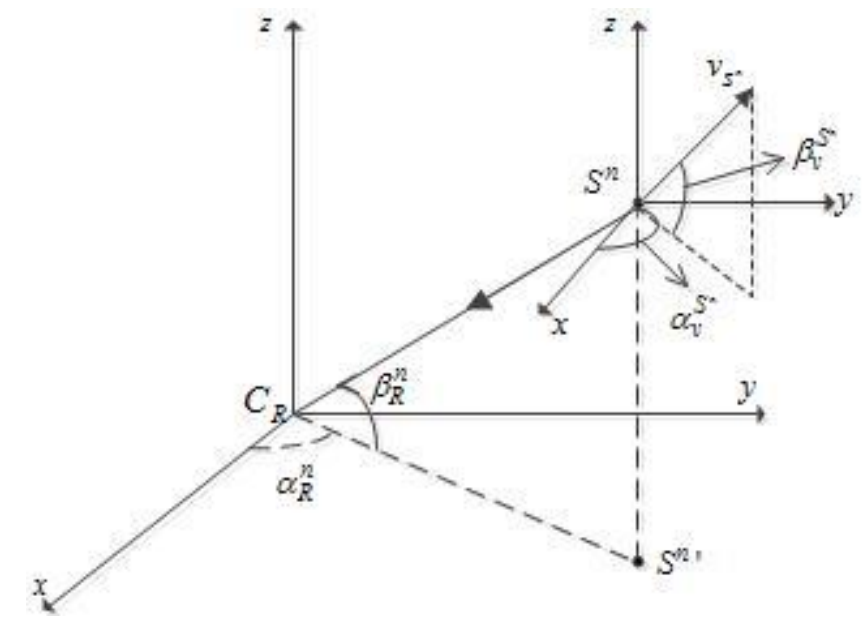

Figure 2. Scattering Path from the Scatterer to the Receiver

The expressions of $f_{n}{ }^{T}, f_{n}{ }^{T S}$ and $f_{n}{ }^{R}$ can be deduced in the same way. Then by the usage of Eq. (3), the total frequency shift $f_{n}$ can be given as Eq. (4), with which the temporal ACF in 3D non-stationary multipath scattering can be given as Eq. (5) [11]. Substituting Eq. (4) into Eq. (5), the temporal ACF of non-stationary 3D multipath scattering channel can be given as Eq. (6). The details of the deduction process from Eq. (5) to Eq. (6) are given as follows:

1) Assuming that the AOD from transmitter $C_{T}$ to the $n$th scatterer $S^{n}$ and the AOA from the $n$th scatterer $S^{n}$ to the receiver $C_{R}$ are independent, the joint PDF $p\left(\alpha_{n}^{T}, \beta_{n}^{T}, \alpha_{n}^{R}, \beta_{n}^{R}\right)$ can be written as $p\left(\alpha_{n}^{T}, \beta_{n}^{T}, \alpha_{n}^{R}, \beta_{n}^{R}\right)=p\left(\alpha_{n}^{T}, \beta_{n}^{T}\right) \times p\left(\alpha_{n}^{R}, \beta_{n}^{R}\right)$.

2) Assuming that the scattered waves departing from transmitter $C_{T}$ to the $n$th scatterer $S^{n}$ transmits with equal probability in the upper semi-sphere, the elemental probability $d p$ of a contributing scattered wave occurring within the element of solid angle $d \Omega$ is $d p=d \Omega /(2 \pi)$, since the total solid angle of a semi- 
sphere is $2 \pi$. Describing the direction of an departing wave in terms of azimuth and elevation AOD $\left(\alpha_{T}^{n}\right.$ and $\left.\beta_{T}^{n}\right)$, the element of solid angle is $d \Omega=\sin \beta_{T}^{n} d_{\beta_{T}^{n}} d_{\alpha_{T}^{n}}$ [18]. The joint PDF of $\alpha_{T}^{n}$ and 3D mobile radio channel statistics can be presented as $p\left(\alpha_{T}^{n}, \beta_{T}^{n}\right)=\sin \beta_{T}^{n} /(2 \pi)$. Similarly, the joint PDF of $\alpha_{R}^{n}$ and $\beta_{R}^{n}$ is $p\left(\alpha_{R}^{n}, \beta_{R}^{n}\right)=\sin \beta_{R}^{n} /(2 \pi)$.

3) Assume scatterers move randomly with equal probability in the whole $3 \mathrm{D}$ sphere space. Since the total solid angle of a sphere is $4 \pi$, the joint PDF of $\alpha_{v}^{s^{n}}$ and $\beta_{v}^{S^{n}}$ can be presented as $p\left(\alpha_{v}^{S_{n}}, \beta_{v}^{S_{n}}\right)=\sin \beta_{v}^{S_{n}} /(4 \pi)$.

4) Substituting these PDFs into Eq. (5), we get the 3D expression of $a(\tau)$ in Eq. (6).

$$
\begin{aligned}
& f_{n}=\frac{v_{T}}{\lambda}\left[\cos \left(\alpha_{T}-\alpha_{T}^{n}\right) \cos \beta_{T}^{n} \cos \beta_{T}+\sin \beta_{T}^{n} \sin \beta_{T}\right]+ \\
& \frac{v_{S^{n}}}{\lambda}\left[\cos \left(\alpha_{v}^{S^{n}}-\alpha_{T}^{n}\right) \cos \beta_{T}^{n} \cos \beta_{v}^{S^{n}}+\sin \beta_{T}^{n} \sin \beta_{v}^{S^{n}}\right]+ \\
& \frac{v_{S^{n}}}{\lambda}\left[\cos \left(\alpha_{v}^{S^{n}}-\alpha_{R}^{n}\right) \cos \beta_{R}^{n} \cos \beta_{v}^{S^{n}}+\sin \beta_{R}^{n} \sin \beta_{v}^{S^{n}}\right]+ \\
& \frac{v_{R}}{\lambda}\left[\cos \left(\alpha_{R}-\alpha_{R}^{n}\right) \cos \beta_{R}^{n} \cos \beta_{R}+\sin \beta_{R}^{n} \sin \beta_{R}\right] \\
& a(\tau)=\iint_{S \times S} \iint_{S \times S} \iint_{S \times S} e^{j 2 \pi f_{n} \tau} \times p\left(\alpha_{v}^{S^{n}}, \beta_{v}^{S^{n}}\right) \times p\left(\alpha_{T}^{n}, \beta_{T}^{n}, \alpha_{R}^{n}, \beta_{R}^{n}\right) \times p\left(v_{s}\right) \times \\
& d_{\alpha_{v}^{s_{n}^{n}}} d_{\beta_{v}^{s_{n}}} d_{\alpha_{\tau}^{n}} d_{\beta_{T}^{n}} d_{\alpha_{R}^{n}} d_{\beta_{R}^{n}} \\
& \left.a(\tau)=\int_{0}^{2 \pi} \int_{0}^{\pi} \int_{0}^{2 \pi} \int_{0}^{\pi / 2} \int_{0}^{2 \pi} \int_{0}^{\pi / 2} e^{j \cdot 2 \pi\left\{\left\{v_{s^{n}}\left[\cos \left(\alpha_{v}^{s^{n}}-\alpha_{\tau}^{n}\right) \cos \beta_{T}^{n} \cos \beta_{v}^{s^{n}}+\sin \beta_{T}^{n} \sin \beta_{v}^{s^{n}}\right] \tau\right.\right.}\right\} \times \\
& e^{j \cdot 2 \pi}\left\{\frac{v_{s^{n}}}{\lambda}\left[\cos \left(\alpha_{v}^{s^{n}}-\alpha_{R}^{n}\right) \cos \beta_{R}^{n} \cos \beta_{v}^{s^{n}}+\sin \beta_{R}^{n} \sin \beta_{v}^{s^{n}}\right] \tau\right\} \\
& \left.e^{j \cdot 2 \pi\left\{\frac{v_{T}}{\lambda}\left[\cos \left(\alpha_{T}-\alpha_{T}^{n}\right) \cos \beta_{T}^{n} \cos \beta_{T}+\sin \beta_{T}^{n} \sin \beta_{T}\right] \tau\right.}\right\} \times \\
& \left.e^{j \cdot 2 \pi\left\{\frac{v_{R}}{\lambda}\left[\cos \left(\alpha_{R}-\alpha_{R}^{n}\right) \cos \beta_{R}^{n} \cos \beta_{R}+\sin \beta_{R}^{n} \sin \beta_{R}\right] \tau\right.}\right\} \times \\
& \frac{\sin \beta_{T}^{n}}{2 \pi} \times \frac{\sin \beta_{R}^{n}}{2 \pi} \times \frac{\sin \beta_{v}^{S^{n}}}{4 \pi} d_{\alpha_{v}^{s^{n}}} d_{\beta_{v}^{s^{n}}} d_{\alpha_{T}^{n}} d_{\beta_{T}^{n}} d_{\alpha_{R}^{n}} d_{\beta_{R}^{n}}
\end{aligned}
$$

Equation (6) is a complicated integration with six dimensions. It is hard to use symbolic integration to give out its exact result, while numerical integration can be adopted to get its numerical solution. The efficiency of classical numerical integration methods, such as Simpson method and trapezoid method, descends rapidly with the increase of dimension. Therefore, to obtain the numerical results of Eq. (6), Monte Carlo integration is suggested, as the efficiency of Monte Carlo integration is not affected by the dimension of integration. However, the accuracy of Monte Carlo integration highly relies on random numbers, then we propose a raytracing based simulation model. 


\section{Ray-Tracing Based Simulation Model}

Considering the scattering scenario shown in Figure 1, a simulation method based on ray-tracing is proposed herein to get the numerical solution of Eq. (6). As the Doppler frequency shift can also be written as $f_{n}=1 /(2 \pi \cdot \Delta t) \cdot \Delta \varphi$ [19], where $\Delta \varphi=2 \pi \Delta l / \lambda$, and $\Delta l$ represents the length difference of the scattering path within $\Delta t$, the complex channel gain in Eq. (1) can be written as

$$
\mu(t)=\sum_{n=1}^{N_{s}} c_{n} e^{j\left(\frac{2 \pi}{\lambda} \Delta l\right)}
$$

Applying the standard ray-tracing method to Equation (7) deduced from Figure 1, the procedure of ray-tracing method to carry out the numerical calculation of Eq. (6) is illustrated in Figure 3.

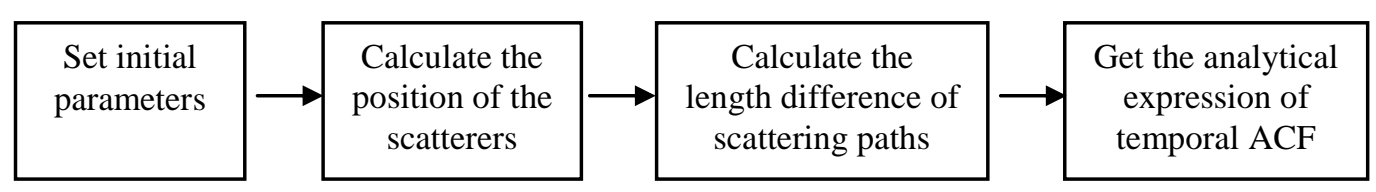

Figure 3. The Procedure of Ray-Tracing Method

The main work procedure can be summarized as follows:

1) Initial parameters setup: the parameters that are necessary for simulation include position for transceivers, carrier frequency $f_{c}$, number of scaterers $N_{s}$, velocity of scatterers $v_{s^{n}}$, velocity direction of scatterer $\left(\alpha_{v}^{s^{n}}, \beta_{v}^{s^{n}}\right)$, velocity of the transmitter $v_{T}$, velocity of receiver $v_{R}$, velocity direction of transmitter $\left(\alpha_{T}, \beta_{T}\right)$ and receiver $\left(\alpha_{R}, \beta_{R}\right)$.

2) Calculate the position of $S^{n}$ at $\Delta t$. For the $n$th scatterer $S^{n}$, the moving distance in a minimal interval $t_{d}$ is $\Delta S=v_{S^{n}} \cdot t_{d}$. Projecting $\Delta S$ onto $\mathrm{x}$-axis, $\mathrm{y}$-axis and z-axis, the three components can be given as $\Delta S_{x}=\Delta S \cdot \cos \beta_{v}^{S^{n}} \cdot \cos \alpha_{v}^{S^{n}}$, $\Delta S_{y}=\Delta S \cdot \cos \beta_{v}^{S^{n}} \cdot \sin \alpha_{v}^{S^{n}}$, and $\Delta S_{z}=\Delta S \cdot \sin \beta_{v}^{S^{n}}$. Supposing $\Delta t=N \times t_{d}$, we can get the ultimate position of $S^{n}$ at $\Delta t$ by accumulating the $N$ components on the initial position at $\mathrm{x}$-axis, $\mathrm{y}$-axis and $\mathrm{z}$-axis, respectively.

3) Calculate the length difference $\Delta l$ of the $n$th scattering path. With the known position of transceivers and the obtained position of $S^{n}$, the distance between $A_{T}$ and $S^{n}\left(\left|A_{T} S^{n}\right|\right)$ and the distance between $S^{n}$ and $A_{T}\left(\left|S^{n} A_{R}\right|\right)$ can be calculated by the usage of Euclidean distance formula. Then the total length of the scattering path at $\Delta t$ can be obtained by adding $\left|A_{T} S^{n}\right|$ and $\left|S^{n} A_{R}\right|$ together $\left|A_{T} A_{R}\right|=\left|A_{T} S^{n}\right|+\left|S^{n} A_{R}\right|$. Assuming that the the initial length of the scattering path at $\Delta t=0$ is $\left|A_{T 0} A_{R 0}\right|$, the length difference of the scattering path at $\Delta t(\Delta t>0)$ can be given as $\Delta l=|| A_{T} A_{R}|-| A_{T 0} A_{R 0} \|$.

4) Obtain the analytical expression for temporal ACF. Substituting $\Delta l$ into Eq. (7), the complex channel gain at $\Delta t$ can be obtained. According to the definition of $\mathrm{ACF}, a(\tau)=\mathrm{E}\left\{\mu^{*}(\mathrm{t}) \mu(\mathrm{t}+\tau)\right\}$, the temporal ACF can be calculated at last.

Instead of using the numerical integration, the proposed ray-tracing based 
simulation model can give out the numerical solution for Eq. (6) from the point of view of geometrical electromagnetic. The model can also simulate $2 \mathrm{D}$ scenario, F2F scenario, and the scenario with different density of scatterers.

\section{Numerical Results}

The initial parameters in simulation are set as follows: the position of transmitter is $(-20,0,0)$, the position of receiver is $(0,0,0), f_{c}=900 \mathrm{M} \mathrm{Hz}, N_{S}=100, \alpha_{T}=\pi / 4$, $\alpha_{R}=0$. Here we assume the transceivers move on the same horizontal plane and set $\beta_{T}=\beta_{R}=0$. These parameters are used for all the following simulation unless otherwise specified.

Figure 4 illustrates the $a(\tau)$ of 2D F2F scenario in [11] by the usage of different method of calculation. The black solid line is the result of Monte Carlo integration. The blue solid line is the result of the proposed ray-tracing based simulation model by setting $\beta_{R}^{n}=0, \beta_{T}^{n}=0$ and $\beta_{v}^{S^{n}}=0$ which degrades our $3 \mathrm{D}$ model into $2 \mathrm{D}$ case. The red scatters is the result of $a(\tau)$ in Figure 5 of [11] under the assumption of uniformly distributed velocity of scatters. It shows that: the ray-tracing based simulation model is an effective method to calculate $a(\tau)$ and can easily apply to the 2D scenario in [11] which serves as a special case of our proposed model.

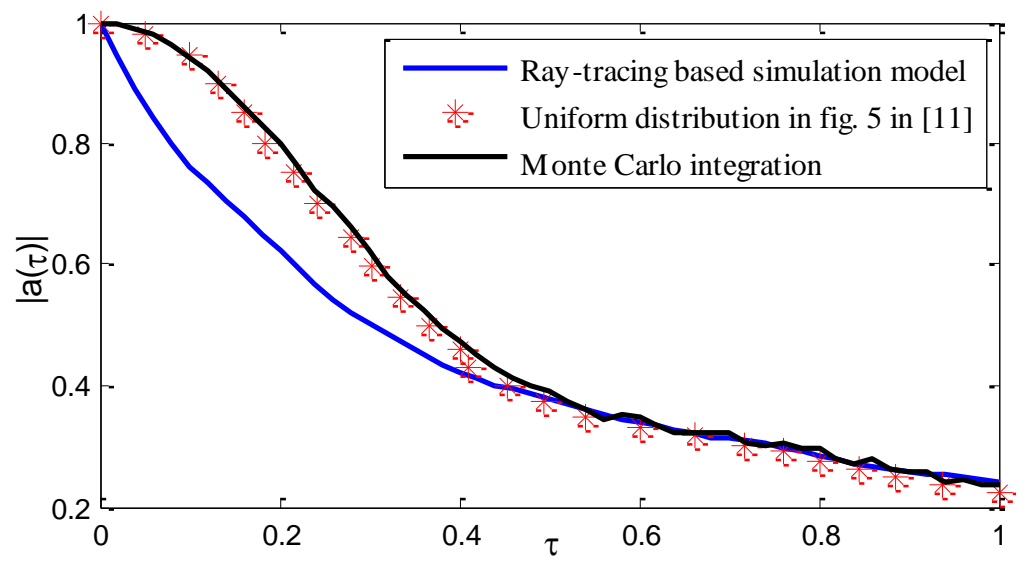

Figure 4. Validation of the Proposed Ray-Tracing Based Simulation Model in Non-Stationary 2D Scenario

Figure 5 illustrates the $a(\tau)$ of $3 \mathrm{D}$ V2V scenario, where $v_{S^{n}}=10 \mathrm{~m} / \mathrm{s}$, $v_{T}=5 \mathrm{~m} / \mathrm{s}, v_{R}=5 \mathrm{~m} / \mathrm{s}, \alpha_{T}=\pi / 6, \alpha_{R}=2 \pi / 3$. Blue dashed line is the result of the proposed ray-tracing based simulation model. The red solid line is the results of $a(\tau)$ in Eq. (6) using Monte Carlo integration. These two curves show a great consistence, which indicates that the ray-tracing based simulation model is an effective and simplified method to obtain the numerical results of $a(\tau)$ in 3D scattering scenario. 


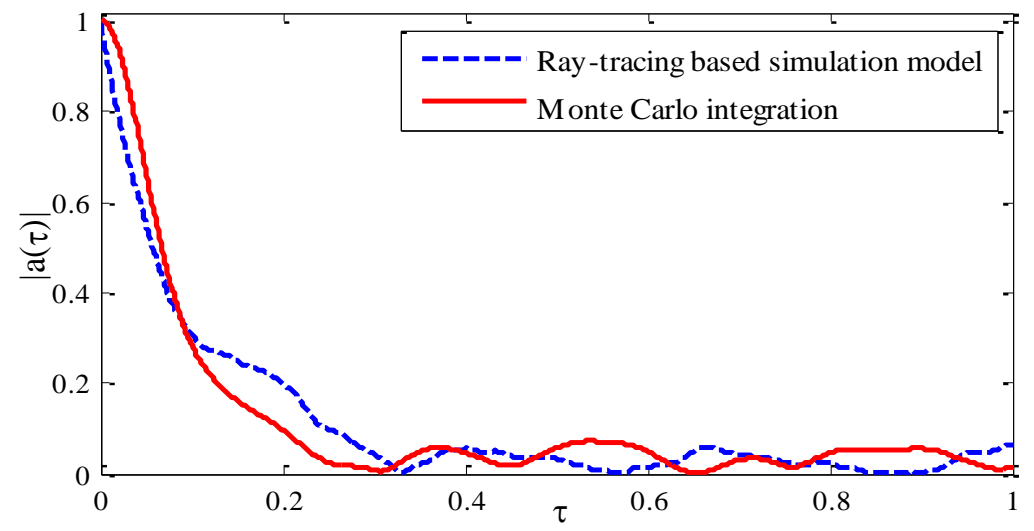

Figure 5. Validation of the Proposed Ray-Tracing Based Simulation Model in Non-Stationary 3D Scenario

Figure 6 illustrates the $a(\tau)$ with different velocities for non-stationary 3D F2F scenario, which reflects the impact of the random movement of scaterers on $a(\tau)$. It shows that: 1) the temporal ACF $a(\tau)$ is sensitive to the velocity of scatters, to be more specific, a little increase of the velocity of scaterers can affect $a(\tau)$ a lot; 2) the temporal ACF $a(\tau)$ attenuates more rapidly with the increase of the velocity of scatterers.

Figure 7 illustrates the $a(\tau)$ under different scatterer velocities for non-stationary 3D V2V scenario, where $v_{T}=10 \mathrm{~m} / \mathrm{s}, v_{R}=20 \mathrm{~m} / \mathrm{s}$, and transceivers move in the same direction by setting $\alpha_{T}=0$ and $\alpha_{R}=0$. It shows that: 1) the temporal ACF $a(\tau)$ attenuates faster with the increase of the velocity of the scatterers; 2) comparing the $a(\tau)$ in Fig. 6 for $v_{s^{n}}=5 \mathrm{~m} / \mathrm{s}$ (3D F2F case), the $a(\tau)$ in Fig. 7 (3D V2V case) also attenuates more faster and shows more fluctuation. This implies that the introduction of moving transceiver may increase the total Doppler shift.

Figure 8 illustrates the $a(\tau)$ under different scatterer densities, where $v_{T}=5 \mathrm{~m} / \mathrm{s}$, $v_{R}=10 \mathrm{~m} / \mathrm{s}, \alpha_{T}=\alpha_{R}=\pi / 6$, and $v_{S^{n}}=5 \mathrm{~m} / \mathrm{s}$. It shows that: $a(\tau)$ attenuates more rapidly and changes more frequently with the increase of the scatterer density.

Rapid attenuation of temporal ACF $a(\tau)$ means the decrease of coherence time and coherence distance. These results together can provide engineers good reference to determine the key parameters of future V2V communication systems.

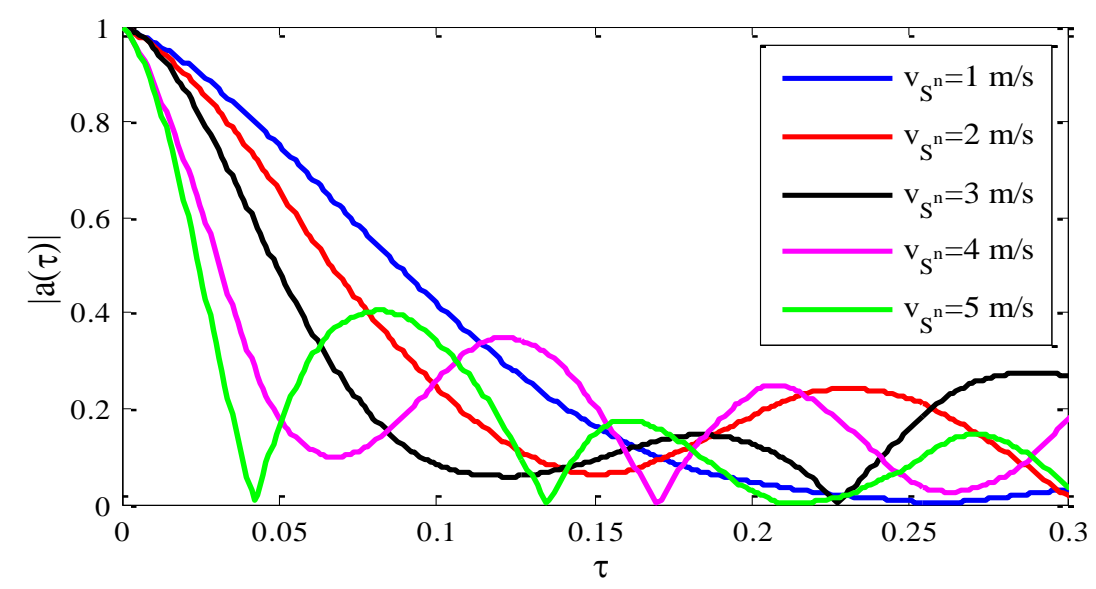

Figure 6. ${ }^{a(\tau)}$ With Different Velocities In 3D F2F Scenario 


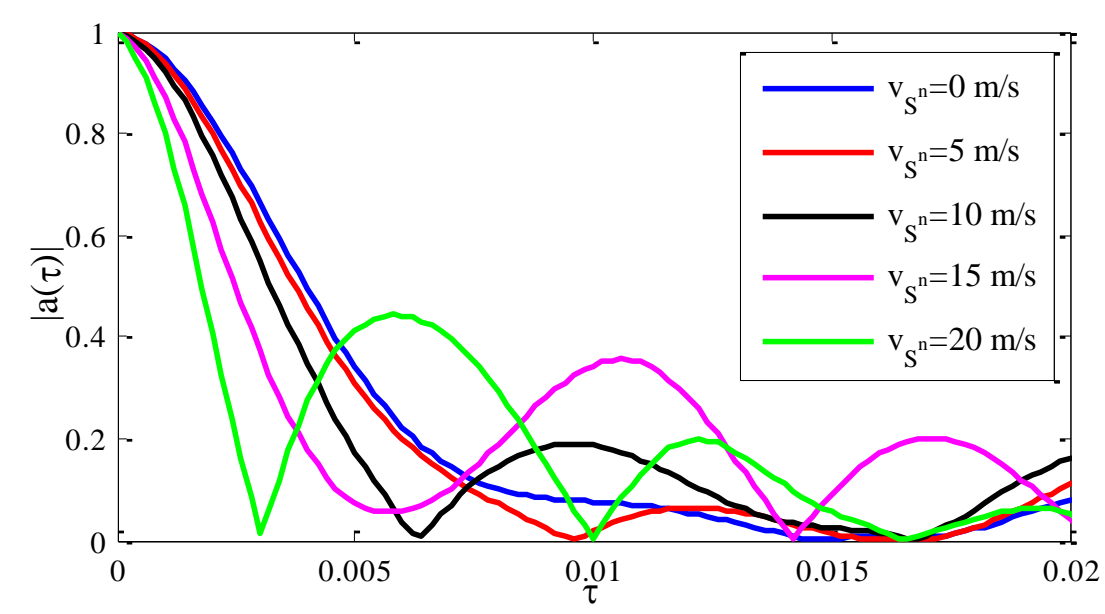

Figure 7. ${ }^{a(\tau)}$ With Different Velocities in 3D V2V Scenario

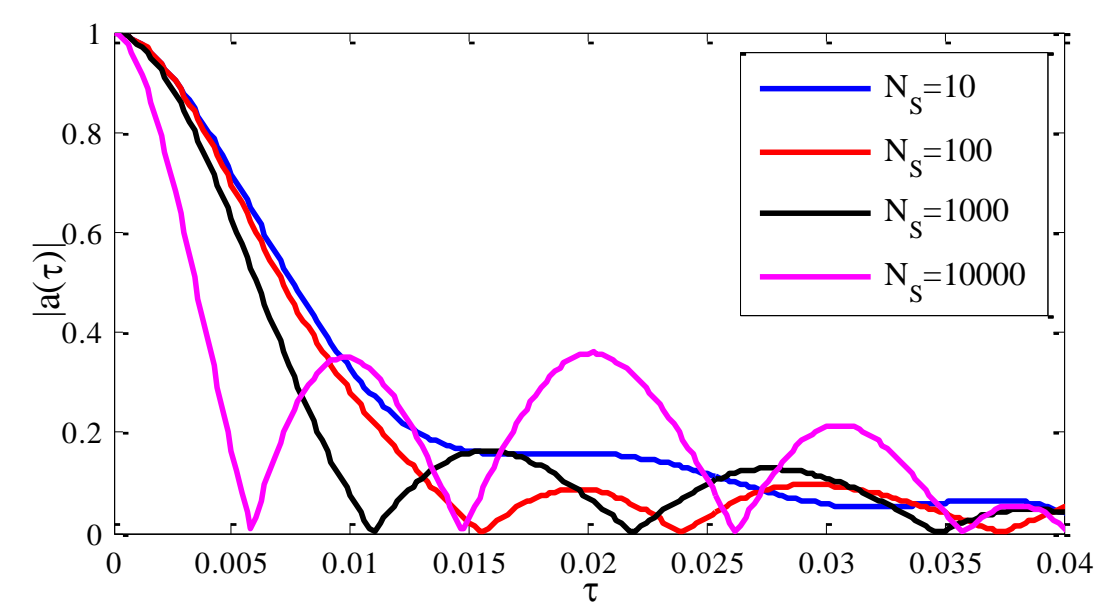

Figure 8. ${ }^{a(\tau)}$ With Different Numbers of Scatterers in 3D V2V scenario

\section{Conclusions}

According to the channel characteristics of $\mathrm{V} 2 \mathrm{~V}$ communication systems, the analytical expression for the temporal autocorrelation function of the non-stationary three dimensional (3D) multipath scattering is derived, where the scatters are in random motion relative to transceivers. The numerical solution of the analytical expression is given by the ray-tracing based simulation model. The analytical expression and the corresponding ray-tracing based methodology has a good scalability: 1) The 2D multipath scattering and the stationary multipath scattering are two typical special cases; 2) the impact of scatterer velocity on the temporal ACF can be discussed for F2F and V2V multipath scattering; 3) the impact of scatterer density on temporal ACF can also be researched. The numerical results show that the temporal ACF would attenuate more rapidly with the increase of the velocity and density of the scatterers. These results allow engineers more appropriate determination on the coherence time and coherence bandwidth for the $3 \mathrm{D} \mathrm{V} 2 \mathrm{~V}$ communication in urban propagation scenario. 


\section{Acknowledgements}

This work was supported by the National Natural Science Foundation of China (NSFC) under Grants 61171089 and the focus project of Chongqing for application and development under Grants cstc2013yykfB0137. The authors would like to thank the anonymous referees whose insightful comments helped us to improve the presentation of the paper.

\section{References}

[1] A. F. Molisch, F. Tufvesson, J. Karedal, and C. F. Mecklenbrauker, "A survey on vehicle-tovehicle propagation channels", IEEE Wireless Communications, vol. 16, (2009), pp. 12-22.

[2] A. Zajic, "Impact of Moving Scatterers on Vehicle-to-Vehicle Narrowband Channel Characteristics", IEEE Transactions on Vehicular Technology, vol, (2014), pp. 1-1

[3] A. Chelli and M. Patzold, "A MIMO Mobile-to-Mobile Channel Model Derived from a Geometric Street Scattering Model", 4th International Symposium on Wireless Communication Systems, Trondheim, (2007) October 17-19, pp. 792-797.

[4] Z. He, W. Chen, W. Zhou, M. Patzold, and A. Chelli, "Modelling of MIMO vehicle-to-vehicle fading channels in T-junction scattering environments", 3rd European Conference on Antennas and Propagation, (2009) March 23-27, pp. 652-656, Berlin.

[5] M. Patzold, B. O. Hogstad, and N. Youssef, "Modeling, analysis, and simulation of MIMO mobile-to-mobile fading channels", IEEE Transactions on Wireless Communications, vol. 7, (2008), pp. 510-520.

[6] C. Xiang, W. Cheng-Xiang, D. I. Laurenson, S. Salous, and A. V. Vasilakos, "An adaptive geometry-based stochastic model for non-isotropic MIMO mobile-to-mobile channels", IEEE Transactions on Wireless Communications, vol. 8, (2009), pp. 4824-4835.

[7] K. N. Le, "On Angle-of-Arrival and Time-of-Arrival Statistics of Geometric Scattering Channels", IEEE Transactions on Vehicular Technology, vol. 58, (2009), pp. 4257-4264.

[8] A. Borhani and M. Patzold, A Unified Disk Scattering Model and Its Angle-of-Departure and Time-of-Arrival Statistics", IEEE Transactions on Vehicular Technology, vol. 62, (2013), pp. 473-485.

[9] V. H. Pham, M. H. Taieb, J. Y. Chouinard, S. Roy, and H. T. Huynh, "On the double Doppler effect generated by scatterer motion", REV J. Electron. Commun, vol. 1, (2011), pp. 30-31.

[10] A. Chelli and M. Patzold, "The Impact of Fixed and Moving Scatterers on the Statistics of MIMO Vehicle-to-Vehicle Channels", IEEE 69th Vehicular Technology Conference, (2009) April 26-29, pp. 1-6, Barcelona.

[11]A. Borhani and M. P̈tzold, "Modeling of Vehicle-to-Vehicle Channels in the Presence of Moving Scatterers", IEEE Vehicular Technology Conference, (2012) September 3-6, pp. 1-5.

[12]A. Borhani and M. Patzold, "Correlation and Spectral Properties of Vehicle-to-Vehicle Channels in the Presence of Moving Scatterers", IEEE Transactions on Vehicular Technology, vol. 62, (2013), pp. 4228-4239.

[13]A. G. Zajic and G. L. Stuber, "Three-Dimensional Modeling, Simulation, and Capacity Analysis of Space-Time Correlated Mobile-to-Mobile Channels", IEEE Transactions on Vehicular Technology, vol. 57, (2008), pp. 2042-2054.

[14] W. Tsan-Ming and T. Tsung-Hua, "Novel 3-D mobile-to-mobile wideband channel model", IEEE Antennas and Propagation Society International Symposium, (2010) July 11-17, pp. 14, Toronto.

[15] P. T. Samarasinghe, T. A. Lamahewa, T. D. Abhayapala, and R. A. Kennedy, "3D Mobile-toMobile wireless channel model", Australian Communications Theory Workshop, (2010) February 2-5, pp. 30 - 34, Canberra, ACT.

[16] M. Riaz, S. J. Nawaz, and N. M. Khan, "3D Ellipsoidal Model for Mobile-to-Mobile Radio Propagation Environments", Wireless personal communications, vol. 72, (2013), pp. 24652479.

[17] M. Patzold, "Mobile Radio Channels", 2nd ed, John Wiley \& Sons, (2011), Chichester.

[18] R. H. Clarke and K. Wee Lin, "3-D mobile radio channel statistics", IEEE Transactions on Vehicular Technology, vol. 46, (1997), pp. 798-799.

[19]G. Zhang and H. Zhang, "Satellite Mobile Communication System", Posts and Telecommunication Press, (2001), Beijing. 


\begin{abstract}
Authors
Xiaoping Zeng, received the B.E., M.S., and Ph.D. degrees in Electrical Engineering from Chongqing University, Chongqing, China in 1982, 1987, and 1996, respectively. He is now a professor and Ph.D. supervisor at the College of Communication Engineering, Chongqing University, China. His research interests include aeronautical mobile communication, wireless communication theory and technology.

Yu Zhou, received her B.E. degree from Chongqing University, Chongqing, China in 2013. She is a master student at the College of Communication Engineering, Chongqing University, China. Her interests include wireless communication systems such as LTE and LTE-Advanced and their applications in intelligent transportation.

Xin Jian, received his B.E. and Ph.D. degree from Chongqing University, Chongqing, China in 2009 and 2014, respectively. He is currently a lecture at the College of Communication Engineering, Chongqing University, China. His interests include statistical learning theory, computational mathematics, network information theory and the next generation mobile communication.

Jie Huang, received his B.E. degree from Chongqing University, Chongqing, China in 2011. He is currently a Ph.D. student of five-years-educational system at the College of Communication Engineering, Chongqing University, China. His interests include internet of things, knapsack problem and cognitive radio.

Jing Li, received her B.E. degree from Henan University, Kaifeng, China in 2014. She is a master student at the College of Communication Engineering, Chongqing University, China. Her interests include the next generation mobile communication systems such as LTE and LTE-Advanced and wireless resource allocation.
\end{abstract}


International Journal of Future Generation Communication and Networking Vol. 8, No. 2 (2015) 